\title{
NOVOS REGISTROS DE HOSPEDEIROS DE MOSCAS-DAS-FRUTAS (DIPTERA: TEPHRITIDAE) PARA O RIO GRANDE DO SUL
}

\author{
Alberto Luiz Marsaro Júnior ${ }^{1}$ \\ ${ }^{1}$ Embrapa Trigo, E-mail: alberto.marsaro@embrapa.br
}

\section{RESUMO}

Visando registrar novos hospedeiros de moscas-das-frutas para o estado do Rio Grande do $\mathrm{Sul}$, foram realizadas coletas aleatórias de frutos de 17 espécies vegetais pertencentes a oito famílias botânicas, nos municípios de Barão de Cotegipe, Herval Grande, Passo Fundo e Quatro Irmãos, entre dezembro de 2010 e outubro de 2012. A partir dos frutos coletados, diretamente nas plantas e caídos no solo, foram obtidas cinco espécies de Anastrepha, Ceratitis capitata e quatro espécies de parasitoides. Anastrepha dissimilis é registrada pela primeira vez em frutos de Passiflora caerulea e Passiflora elegans. Anastrepha pseudoparallela é registrada pela primeira vez em frutos de P. elegans. Anastrepha pickeli é registrada pela primeira vez em frutos de Manihot grahamii e também pela primeira vez no Rio Grande do Sul.

Palavras-chave: Anastrepha, Ceratitis capitata, distribuição geográfica, parasitoides

\section{NEW RECORDS OF FRUIT FLIES HOSTS (DIPTERA: TEPHRITIDAE) IN RIO GRANDE DO SUL STATE, BRAZIL}

\begin{abstract}
Aiming at recording new hosts of fruit flies to the state of Rio Grande do Sul, we performed random samples of fruits of 17 plant species from eight botanical families in the municipalities of Barão de Cotegipe, Herval Grande, Passo Fundo and Quatro Irmãos, from December 2010 to October 2012. From the fruits collected, directly on plants and fallen on the ground, we obtained five species of Anastrepha, Ceratitis capitata and four species of parasitoids. Anastrepha dissimilis is recorded in fruits of Passiflora caerulea and Passiflora elegans for the first time. Also, Anastrepha pseudoparallela is recorded in fruits of P. elegans for the first time. Anastrepha pickeli is first recorded in fruits of Manihot grahamii and also for the first time in Rio Grande do Sul.
\end{abstract}

Keywords: Anastrepha, Ceratitis capitata, geographical distribution, parasitoids

\section{INTRODUÇÃO}

As moscas-das-frutas (Diptera: Tephritidae) estão entre as principais pragas em todo o mundo, devido aos seus impactos econômicos diretos e às severas restrições quarentenárias impostas por muitos países para evitarem as suas entradas (ALUJA, 1994; ALUJA \& MANGAN, 2008). No Brasil, 115 espécies de Anastrepha já foram assinaladas (ZUCCHI, 2008a). Destas, seis são particularmente importantes do ponto de vista econômico: Anastrepha striata (Schiner), Anastrepha obliqua (Macquart), Anastrepha fraterculus (Wiedemann), Anastrepha grandis (Macquart), Anastrepha pseudoparallela (Loew) e Anastrepha zenildae Zucchi (Uramoto \& Zucchi, 2009). Para o estado do Rio Grande do Sul são registradas 16 espécies de Anastrepha 
(ZUCCHI, 2008a), além de Ceratitis capitata (Wiedemann) (ZUCCHI, 2008b), espécie considerada a mais nociva entre os tefritídeos, pois causa mais prejuízos do que qualquer outra, especialmente por ser a mais cosmopolita e invasora de todas (SILVA et al., 2011a). No Brasil, C. capitata já foi registrada em 22 dos 26 estados (ZUCCHI, 2008b). Quanto aos parasitoides, no Rio Grande do Sul, são conhecidas sete espécies associadas a moscas do gênero Anastrepha (ZUCCHI, 2008a; CRUZ et al., 2011).

A relação entre as espécies de Anastrepha e seus frutos hospedeiros no Brasil ainda é pouco conhecida, sendo os estudos mais focados nas espécies de importância econômica (ZUCCHI, 2007, 2008a). No Brasil, as espécies A. fraterculus e $C$. capitata são as que possuem os maiores números de hospedeiros conhecidos, 97 e 84, respectivamente (ZUCCHI, 2008a, 2008b). Até o momento atual, as espécies de Anastrepha e C. capitata possuem poucos registros de hospedeiros no Rio Grande do Sul (ZUCCHI, 2008a, 2008b; NUNES et al., 2012; SAVARIS et al., 2013). Neste trabalho são feitos novos registros de frutos hospedeiros de espécies de Anastrepha e $C$. capitata para o Estado.

\section{MATERIAL E MÉTODOS}

Para conhecer a associação entre as espécies de Anastrepha e C. capitata com suas plantas hospedeiras foram realizadas coletas aleatórias de frutos potencialmente hospedeiros, entre dezembro de 2010 e outubro de 2012, principalmente nos meses de janeiro a abril, em quatro municípios do Rio Grande do Sul, com poucos estudos sobre moscas-das-frutas: Barão de Cotegipe (273' $\mathrm{S}, 52^{\circ} 24^{\prime} \mathrm{W}$ ) (uma coleta), Herval Grande $\left(27^{\circ} 25^{\prime} \mathrm{S}, 52^{\circ} 32^{\prime} \mathrm{W}\right)$ (uma coleta), Passo Fundo (28 $13^{\prime}$ 'S, 52 $26^{\circ}$ 'W) (16 coletas, média de duas coletas/mês) e Quatro Irmãos $\left(27^{\circ} 50^{\prime} \mathrm{S}, 52^{\circ} 21^{\prime} \mathrm{W}\right)$ (uma coleta). Foram coletados frutos maduros diretamente das plantas e caídos no solo, de 17 espécies vegetais, pertencentes a oito famílias botânicas. Os frutos foram acondicionados em caixas de isopor e conduzidos ao Laboratório de Entomologia da Embrapa Trigo, em Passo Fundo, RS. No laboratório, os frutos foram contados, pesados e dispostos em bandejas de plástico com tela, sobre uma camada de vermiculita umedecida, e mantidos em temperatura ambiente. Diariamente, durante 20 dias, o material contido nas bandejas foi examinado e os pupários retirados e transferidos para frascos de plástico transparentes com tela, contendo uma fina camada de vermiculita umedecida, mantidos em temperatura ambiente até a emergência de adultos de moscas e parasitoides, à semelhança de Marsaro Júnior et al. (2011a).

As fêmeas de Anastrepha foram identificadas por caracteres taxonômicos presentes no acúleo (previamente extrovertido), corpo e asa, com base na chave dicotômica descrita por Zucchi (2000). Os exemplares da mosca $C$. capitata foram identificados, por caracteres morfológicos de seu corpo, segundo chave descrita por Zucchi (2000). Os parasitoides foram identificados por caracteres taxonômicos presentes no corpo, sendo os da família Braconidae com base na chave dicotômica descrita por Canal \& Zucchi (2000) e os da família Figitidae pela chave descrita por Guimarães et al. (2000). Todas as identificações foram realizadas com o auxílio de microscópio estereoscópio e microscópio biológico. Voucher specimens foram depositados na coleção da Embrapa Amapá.

A partir dos dados coletados foram calculados os índices de infestação dos frutos (número de pupários/fruto e número de pupários/kg de fruto) por moscas-das-frutas e as porcentagens de parasitismo (número de parasitoides emergidos a partir de um número conhecido de pupários). 


\section{RESULTADOS E DISCUSSÃO}

Foram coletadas 808 fêmeas de Anastrepha e duas de $C$. capitata nas amostragens de frutos nativos e exóticos ao longo de 23 meses. Foram identificadas cinco espécies de Anastrepha: A. fraterculus,
Anastrepha barbiellinii Lima, Anastrepha pickeli Lima, Anastrepha dissimilis Stone e Anastrepha pseudoparallela (Loew), pertencentes a três grupos de espécies, segundo a classificação de Norrbom et al. (1999) (Tabela 1).

Tabela 1. Espécies de Anastrepha obtidas de frutos nativos e exóticos em quatro municípios do estado do Rio Grande do Sul entre dezembro de 2010 e outubro de 2012.

\begin{tabular}{ll}
\hline Grupos & \multicolumn{1}{c}{ Espécies } \\
\hline fraterculus & A. barbiellinii Lima \\
pseudoparallela & A. fraterculus (Wiedemann) \\
Spatulata & A. dissimilis Stone \\
& A. pseudoparallela (Loew) \\
& A. pickeli Lima \\
\hline
\end{tabular}

Anastrepha barbiellinii ocorreu em frutos de Pereskia aculeata Mill. (Cactaceae) (Tabela 2). Esse tefritídeo tem sido registrado neste mesmo hospedeiro para o estado de Minas Gerais (MARSARO JÚNIOR et al., 2011b) e Santa Catarina (GARCIA \& NORRBOM, 2011). Agora, A. barbiellinii passa também a ser reportada em $P$. aculeata para o Rio Grande do Sul.

Anastrepha dissimilis foi obtida em frutos de Passiflora caerulea L. e Passiflora elegans Mast. (Passifloraceae) (Tabela 2). Esse tefritídeo já havia sido associado a Passiflora sp. (ZUCCHI, 2008a) e Passiflora edulis (GARCIA \& NORRBOM, 2011), mas as associações desta mosca com frutos de $P$. caerulea e $P$. elegans são novos registros para o Brasil.

Anastrepha fraterculus foi registrada em frutos de Annona rugulosa (Schltdl.) H. Rainer (Annonaceae), Diospyros kaki L. f. (Ebenaceae), Acca sellowiana (O. Berg) Burret, Campomanesia guazumifolia (Cambess.) O. Berg, Campomanesia xanthocarpa O. Berg, Eugenia involucrata DC., Eugenia pyriformis Cambess., Eugenia uniflora L., Myrcianthes pungens (O. Berg)
D. Legrand, Psidium cattleianum Sabine, Psidium guajava L. (Myrtaceae) e Prunus persica (L.) Batsch (Rosaceae) (Tabela 2). Portanto, A. fraterculus foi a espécie predominante, sendo associada a 12 das 17 espécies vegetais avaliadas. Esse tefritídeo já havia sido associado a A. rugulosa, $C$. guazumifolia e M. pungens em Santa Catarina (GARCIA \& NORRBOM, 2011), sendo inéditas essas associações para o Rio Grande do Sul.

Anastrepha pickeli ocorreu em frutos de Manihot grahamii Hook. (Euphorbiaceae) (Tabela 2). Esse tefritídeo é relatado para os estados do Amapá, Amazonas, Bahia, Espírito Santo, Maranhão, Mato Grosso do Sul, Mato Grosso, Minas Gerais, Pará, Pernambuco, Piauí, Rio de Janeiro, Rio Grande do Norte, Roraima, São Paulo, Santa Catarina e Tocantins (ZUCCHI, 2008a), e tem como hospedeiros conhecidos frutos de Manihot esculenta Crantz (Euphorbiaceae) e Quararibea turbinata (Swartz) Poiret (Bombacaceae). A ocorrência de A. pickeli no estado do Rio Grande do Sul constitui novo registro, assim como seu hospedeiro $(M$. grahamii). 
Tabela 2. Hospedeiros de Anastrepha spp. e Ceratitis capitata e parasitoides associados em quatro municípios do Rio Grande do Sul, Barão de Cotegipe (BC), Herval Grande (HG), Passo Fundo (PF) e Quatro Irmãos (QI).

\begin{tabular}{|c|c|c|c|c|c|c|c|c|c|}
\hline \multirow{2}{*}{$\begin{array}{l}\text { Família } \\
\text { Nome científico - Nome vernacular }\end{array}$} & \multirow{2}{*}{ Municípios } & \multirow{2}{*}{$\begin{array}{c}\text { Frutos } \\
(\mathbf{n})\end{array}$} & \multirow{2}{*}{$\begin{array}{c}\text { Massa } \\
(\mathbf{k g})\end{array}$} & \multirow{2}{*}{$\begin{array}{l}* \mathbf{P} \\
(\mathbf{n})\end{array}$} & \multicolumn{2}{|c|}{ Infestação } & \multirow{2}{*}{$\begin{array}{c}* * \mathbf{P P} \\
(\%)\end{array}$} & \multirow{2}{*}{ Tephritidae } & \multirow{2}{*}{ Hymenoptera } \\
\hline & & & & & P/Fruto & $\mathbf{P} / \mathrm{kg}$ & & & \\
\hline $\begin{array}{l}\text { Annonaceae } \\
{ }^{1} \text { Annona rugulosa-Araticum }\end{array}$ & $\mathrm{PF}$ & 9 & 0,47 & 19 & 2,11 & 39,67 & 10,53 & A. fraterculus $(12+, 5 \AA)$ & D. areolatus (2) \\
\hline $\begin{array}{l}\text { Cactaceae } \\
{ }^{1} \text { Pereskia aculeata - Ora-pro-nóbis }\end{array}$ & HG & 30 & 0,25 & 26 & 0,87 & 104,00 & 7,69 & A. barbiellinii $(14 \odot, 8$ ) & D. brasiliensis (2) \\
\hline $\begin{array}{l}\text { Ebenaceae } \\
\text { Diospyros kaki-Caqui }\end{array}$ & $\mathrm{PF}$ & 20 & 1,47 & 19 & 0,95 & 12,93 & 0 & $\begin{array}{l}\text { A. fraterculus }\left(3 \circ, 9{ }^{\lambda}\right) \\
\text { C. capitata }\left(1+, 4 \bigcirc^{\lambda}\right)\end{array}$ & \\
\hline $\begin{array}{l}\text { Euphorbiaceae } \\
{ }^{2} \text { Manihot grahamii - Mandioca-brava }\end{array}$ & $\mathrm{BC}$ & 50 & 0,19 & 3 & 0,06 & 15,34 & 0 & ${ }^{3}$ A. pickeli $\left(3 \stackrel{+}{ }, 0{ }^{\top}\right)$ & \\
\hline $\begin{array}{l}\text { Malpighiaceae } \\
{ }^{1} \text { Malpighia emarginata - Acerola }\end{array}$ & $\mathrm{PF}$ & 156 & 0,96 & 3 & 0,02 & 3,13 & 0 & 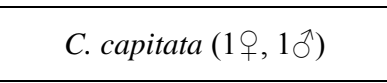 & \\
\hline $\begin{array}{l}\text { Myrtaceae } \\
\text { Acca sellowiana - Goiaba-serrana }\end{array}$ & $\mathrm{PF}$ & 49 & 0,90 & 736 & 15,02 & 817,78 & 12,23 & A. fraterculus $\left(210 \bigcirc, 242{ }^{\Uparrow}\right)$ & $\begin{array}{c}\text { A. pelleranoi }(24) \\
\text { D. areolatus }(64) \\
\text { D. brasiliensis }(2)\end{array}$ \\
\hline${ }^{1}$ Campomanesia guazumifolia - Sete capotes & $\mathrm{PF}$ & 63 & 0,65 & 60 & 0,95 & 92,31 & 0 & A. fraterculus $\left(22+, 16{ }^{\wedge}\right)$ & \\
\hline Campomanesia xanthocarpa - Guabiroba & $\mathrm{PF}$ & 90 & 0,41 & 42 & 0,47 & 102,44 & 0 & A. fraterculus $(20 \bigcirc, 18 \AA)$ & \\
\hline Eugenia involucrata - Cerejeira & $\mathrm{PF}$ & 100 & 0,26 & 110 & 1,10 & 423,08 & 0 & A. fraterculus $\left(59 \bigcirc, 40 \bigcirc^{\wedge}\right)$ & \\
\hline \multirow{2}{*}{ Eugenia pyriformis - Uvaia } & \multirow{2}{*}{$\mathrm{PF}$} & 166 & 1,53 & 220 & 1,33 & 143,79 & 0,45 & A. fraterculus $(71+9,95 \AA)$ & D. areolatus (1) \\
\hline & & 33 & 0,19 & 24 & 0,73 & 123,08 & 4,17 & A. fraterculus $(15 \bigcirc, 7 \hat{)})$ & A. pelleranoi (1) \\
\hline Eugenia uniflora - Pitanga & $\mathrm{PF}$ & 100 & 0,16 & 20 & 0,20 & 125,00 & 0 & A. fraterculus $\left(4+, 6 \bigcirc^{\Uparrow}\right)$ & \\
\hline${ }^{1}$ Myrcianthes pungens - Guabiju & $\mathrm{PF}$ & 130 & 0,65 & 173 & 1,33 & 266,15 & 0,58 & A. fraterculus $(51+, 90 \AA)$ & D. areolatus (1) \\
\hline Psidium cattleianum - Araçá-vermelho & $\mathrm{PF}$ & 31 & 0,22 & 30 & 0,97 & 136,36 & 0 & A. fraterculus $\left(11+, 18 \delta^{\uparrow}\right)$ & \\
\hline $\begin{array}{l}\text { Psidium guajava } \mathrm{L} . \\
\text { Goiaba }\end{array}$ & $\mathrm{PF}$ & 36 & 2,52 & 134 & 3,72 & 53,17 & 2,24 & 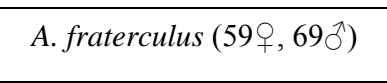 & D. areolatus (3) \\
\hline $\begin{array}{l}\text { Passifloraceae } \\
{ }^{2} \text { Passiflora caerulea - Maracujá-azul }\end{array}$ & $\mathrm{PF}$ & 50 & 0,74 & 40 & 0,80 & 54,05 & 0 & A. dissimilis $\left(15 \bigcirc, 21 ठ^{\Uparrow}\right)$ & \\
\hline \multirow[b]{2}{*}{${ }^{2}$ Passiflora elegans - Maracujá-de-estalo } & $\mathrm{PF}$ & 49 & 0,44 & 2 & 0,04 & 4,55 & 0 & A. dissimilis $(2+, 0 \widehat{ })$ & \\
\hline & QI & 50 & 0,52 & 31 & 0,62 & 59,62 & 0 & 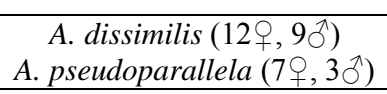 & \\
\hline $\begin{array}{l}\text { Rosaceae } \\
\text { Prunus persica - Pêssego }\end{array}$ & $\mathrm{PF}$ & 41 & 2,87 & 661 & 16,12 & 230,31 & 20,88 & A. fraterculus $(218 \bigcirc, 199 \widehat{)})$ & $\begin{array}{c}\text { A. pelleranoi }(113) \\
\text { D. areolatus }(1) \\
\text { D. brasiliensis } \\
(19) \\
\text { Opius bellus }(5)\end{array}$ \\
\hline
\end{tabular}

*P = número de pupários; **PP = percentual de parasitismo (número de parasitoides emergidos a partir de um número conhecido de pupários).

${ }^{1}$ Novo registro de hospedeiro para o Rio Grande do Sul; ${ }^{2}$ Novo registro de hospedeiro para o Brasil; ${ }^{3}$ Novo registro da espécie para o Rio Grande do Sul. 
Anastrepha pseudoparallela emergiu de frutos de $P$. elegans (Passifloraceae) (Tabela 2). A ocorrência desta mosca em frutos de $P$. elegans também é novo registro. Anteriormente, A. pseudoparallela havia sido registrada em frutos de Mangifera indica L. (Anacardiaceae), Passiflora alata Dryander, Passiflora edulis Sims, Passiflora quadrangularis L. (Passifloraceae) e $P$. guajava (Myrtaceae) (URAMOTO et al., 2004; Zucchi, 2008a; Garcia \& Norrbom, 2011).

Ceratitis capitata ocorreu associada a frutos de D. kaki (Ebenaceae) e Malpighia emarginata Sessé \& Moc. ex DC. (Malpighiaceae) (Tabela 2). Esse tefritídeo já havia sido associado a $M$. emarginata em São Paulo (RAGA et al., 2011), mas para o Rio Grande do Sul essa associação é inédita.

Acca sellowiana e Prunus persica foram os hospedeiros que apresentaram os maiores índices de infestação (número de pupários/fruto), 15 e 16, respectivamente (Tabela 2). Para A. sellowiana, Gatelli et al. (2008) obtiveram 2,6 pupários/fruto, enquanto Garcia \& Norrbom (2011) encontraram 6,2 pupários/fruto. Para $P$. persica, Garcia \& Norrbom (2011) obtiveram 1,8 pupário/kg, enquanto Montes et al. (2011) encontraram o valor máximo de 8,8 moscas/fruto. Essas diferenças de resultados podem estar relacionadas à disponibilidade dos frutos hospedeiros nas ocasiões das amostragens, às diferentes épocas, localidades e regiões das coletas. Gatelli et al. (2008), por exemplo, em estudo conduzido com mirtáceas nas cidades de Montenegro e Harmonia, no Rio Grande do Sul, constaram que dentre os hospedeiros amostrados, goiaba, goiaba-serrana, araçá-vermelho, guabiroba e pitanga, a goiaba foi a que apresentou a maior infestação (cerca de 8 pupários/fruto). No presente trabalho, para este mesmo hospedeiro (goiaba), foram obtidos cerca de 4 pupários/fruto.

Considerando a variável pupários/kg de fruto, a goiaba-serrana foi a que apresentou a maior infestação (cerca de 818 pupários $/ \mathrm{kg}$ ) (Tabela 2). Esse índice, embora seja elevado, ainda é inferior ao obtido por Silva et al. (2011b) em frutos de Brosimum potabile (Moraceae), que foi de 1.048 pupários $/ \mathrm{kg}$.

Foram obtidas as seguintes espécies de parasitoides (Hymenoptera) associadas à espécie A. fraterculus: Doryctobracon areolatus (Szépligeti), Doryctobracon brasiliensis (Szépligeti) e Opius bellus Gahan (todos Braconidae) e Aganaspis pelleranoi (Brèthes) (Figitidae). O parasitoide associado a A. barbiellinii foi $D$. brasiliensis. Por outro lado, nenhum desses parasitoides foi associado a $C$. capitata (Tabela 2). Nunes et al. (2012) obtiveram três espécies associadas a $A$. fraterculus, $D$. areolatus, $O$. bellus e $A$. pelleranoi, mas também não registraram nenhuma espécie associada a $C$. capitata, em frutos de caqui.

A maior taxa de parasitismo observada de A. fraterculus foi de $20,8 \% \mathrm{em}$ frutos de pêssego, $P$. persica (Tabela 2). Num outro estudo, conduzido por Nunes et al. (2012), os autores registraram 1,2\% para esse mesmo tefritídeo e hospedeiro. Visto que, no presente trabalho, os frutos foram coletados de um pomar onde não se aplicava inseticidas, provavelmente essa prática contribuiu para a preservação dos parasitoides e pela elevada taxa de parasitismo observada.

Sugere-se que levantamentos mais amplos e intensos de hospedeiros de Anastrepha spp. e C. capitata sejam realizados no Rio Grande do Sul, tanto em áreas de cultivos agrícolas quanto em áreas pouco alteradas, de forma a contribuir para o conhecimento da ecologia desses tefritídeos e de seus parasitoides.

\section{AGRADECIMENTOS}

Ao Dr. Jorge Anderson Guimarães pela identificação dos exemplares de Aganaspis pelleranoi. Ao Conselho Nacional de Desenvolvimento Científico e Tecnológico - CNPq pela bolsa de 
Produtividade em Pesquisa concedida a Ricardo Adaime e pela bolsa de Doutorado concedida para Marcoandre Savaris (proc. 142105/2012-0) e de Mestrado concedida para Silvana Lampert (proc. 132310/2012-0).

\section{REFERÊNCIAS BIBLIOGRÁFICAS}

ALUJA, M. 1994. Bionomics and management of Anastrepha. Annual Review of Entomology, Palo Alto, v. 39, p. 155-178.

ALUJA, M.; MANGAN, R. L. 2008. Fruit Fly (Diptera: Tephritidae) Host Status Determination: Critical Conceptual, Methodological, and Regulatory Considerations. Annual Review of Entomology, Palo Alto, v. 53, p. 473-502. CANAL, N. A.; ZUCCHI, R. A. 2000. Parasitóides - Braconidae. In: Malavasi, A. \& Zucchi, R.A. (eds.). Moscas-dasfrutas de importância econômica no Brasil: conhecimento básico e aplicado. Ribeirão Preto: Holos, p. 119-126.

CRUZ, P. P.; NEUTZLING, A. S.; GARCIA, F. R. M. 2011. Primeiro registro de Trichopria anastrephae, parasitoide de moscas-das-frutas, no Rio Grande do Sul. Ciência Rural, Santa Maria, v. 41, n. 8, p. 1297-1299.

GARCIA, F. R. M.; NORRBOM, A. L. 2011.Tephritoid flies (Diptera, Tephritoidea) and their plants hosts from the state of Santa Catarina in Southern Brazil. Florida Entomologist, Gainesville, v. 94, n. 2, p. 151-157.

GATTELLI, T.; SILVA, F. F.; MEIRELLES, R. N.; REDAELLI, L. R.; SOGLIO, F. K. D. 2008. Moscas frugívoras associadas a mirtáceas e laranjeira "Céu" na região do Vale do Rio Caí, Rio Grande do Sul, Brasil. Ciência Rural, Santa Maria, v. 38, n. 1, p. 236-239.

GUIMARÃES, J. A.; DIAZ, N. B.; ZUCCHI, R. A. Parasitóides - Figitidae (Eucoilinae). In: Malavasi, A. \& Zucchi, R.A. (eds.). Moscas-das-frutas de importância econômica no Brasil: conhecimento básico e aplicado. Ribeirão Preto: Holos, p. 127-134.

MARSARO JÚNIOR, A. L.; ADAIME, R.; RONCHI-TELES, B.; LIMA, C. R.; PEREIRA, P. R. V. S. 2011a. Anastrepha species (Diptera: Tephritidae), their hosts and parasitoids in the extreme north of Brazil. Biota Neotropica, Campinas, v. 11, n. 4, p. 117-124.

MARSARO JÚNIOR, A. L.; SOUZAFILHO, M. F.; SILVA, R. A.; STRIKIS, P. C. 2011b. First report of natural infestation of Pereskia aculeata MILL. (Cactaceae) by Ceratitis capitata (Wiedemann) (Diptera: Tephritidae) in Brazil. Revista de Agricultura, Piracicaba, v. 86, n. 2, p. 151-154.

MONTES, S. M. N. M.; RAGA, A.; BOLIANI, A. C.; SANTOS, P. C. 2011. Dinâmica populacional e incidência de moscas-das-frutas e parasitoides em cultivares de pessegueiros (Prunus persica L. Batsch) no município de Presidente Prudente-SP. Revista Brasileira de Fruticultura, Jaboticabal, v. 33, n. 2, p. 402-411.

NORRBOM, A. L.; ZUCCHI, R. A.; HERNÁNDEZ-ORTIZ, V. 1999. Phylogeny of the genera Anastrepha and Toxotrypana (Trypetinae: Toxotripanini) based on morphology. In: Norrbom, A. L. \& Aluja, M. (eds.). Fruit flies (Tephritidae): phylogeny and evolution of behavior. Boca Raton: CRC Press, p. 299-342.

NUNES, A. M.; MÜLLER, F. A.; GONÇALVES, R. S.; GARCIA, M. S.; COSTA, V. A.; NAVA, D. E. Moscas frugívoras e seus parasitoides nos municípios de Pelotas e Capão do Leão, Rio Grande do Sul, Brasil. 2012. Ciência Rural, Santa Maria, v. 42, n. 1, p. 6-12.

RAGA, A.; SOUZA-FILHO, M. F.; MACHADO, R. A.; SATO, M. E.; SILOTO, R. C. 2011. Host ranges and infestation indices of fruit flies 
(Tephritidae) and lance flies (Lonchaeidae) in São Paulo state, Brazil. Florida Entomologist, Gainesville, v. 94, n. 4, p. 787-794.

SAVARIS, M.; LAMPERT, S.; MARSARO JÚNIOR, A. L.; ADAIME, R.; SOUZAFILHO, M. F. 2013. First record of Anastrepha fraterculus and Ceratitis capitata (Diptera, Tephritidae) on Arecaceae in Brazil. Florida Entomologist, Gainesville, v. 96, n. 4, p. 1597-1599.

SILVA, R. A.; LEMOS, W. P.; ZUCCHI, R. A. 2011a. Ocorrência e hospedeiros de Ceratitis capitata na Amazônia brasileira In: SILVA, R. A.; LEMOS, W. P.; ZUCCHI, R. A. (Eds.). Moscas-dasfrutas na Amazônia brasileira: diversidade, hospedeiros e inimigos naturais. Macapá: Embrapa Amapá, p. 197-204.

SILVA, R. A.; DEUS, E. G.; PEREIRA, J. D. B.; JESUS, C. R.; SOUZA-FILHO, M. F.; ZUCCHI, R. A. 2011b. Conhecimento sobre moscas-das-frutas no Estado do Amapá. In: SILVA, R. A.; LEMOS, W. P.; ZUCCHI, R. A. (Eds.). Moscas-dasfrutas na Amazônia brasileira: diversidade, hospedeiros e inimigos naturais. Macapá: Embrapa Amapá, p. 223-236.

URAMOTO, K.; ZUCCHI, R. A. 2009. Taxonomia de espécies de Anastrepha (Diptera: Tephritidae). In: MALAVASI, A.; VIRGINIO, J. (Eds.). Biologia, Monitoramento e Controle: V Curso Internacional de Capacitação em Moscasdas-frutas. Juazeiro: MOSCAMED, p. 711.

URAMOTO, K.; WALDER, J. M. M.; ZUCCHI, R. A. 2004. Biodiversidade de moscas-das-frutas do gênero Anastrepha (Diptera, Tephritidae) no campus da ESALQ-USP, Piracicaba, São Paulo. Revista Brasileira de Entomologia, São Paulo, v. 48, n. 3, p. 409-414.
ZUCCHI, R. A. 2000. Taxonomia. In: MALAVASI, A. \& ZUCCHI, R. A. (eds.). Moscas-das-frutas de importância econômica no Brasil: conhecimento básico e aplicado. Ribeirão Preto: Holos, p. 13-24.

ZUCCHI, R. A. 2007. Diversidad, distribución y hospederos del género Anastrepha en Brasil. In: HERNÁNDEZORTIZ, V. (Ed.). Moscas de la fruta en Latinoamérica (Diptera: Tephritidae): Diversidad, biologia y manejo. Distrito Federal, México: S y G editores, p. 77-100. ZUCCHI, R. A. 2008a. Fruit flies (Diptera: Tephritidae) in Brazil - Anastrepha species their host plants and parasitoids. Disponível em: http://www.lea.esalq.usp.br/anastrepha/. Acesso em: 17 de dezembro de 2013.

ZUCCHI, R. A. 2008b. Fruit flies (Diptera: Tephritidae) in Brazil - Hosts and parasitoids of the Mediterranean fruit fly. Disponível em: http://www.lea.esalq.usp.br/ceratitis/. Acesso em: 17 de dezembro de 2013.

Recebido em: 08/1/2014 Aceito para publicação em: 02/4/2014 